\title{
Zonisamide-loaded triblock copolymer nanomicelles as a novel drug delivery system for the treatment of acute spinal cord injury
}

This article was published in the following Dove Press journal:

International Journal of Nanomedicine

29 March 2017

Number of times this article has been viewed

\author{
JingLun $\mathrm{Li}^{\prime}$ \\ Jiaojiao Deng² \\ JinXian Yuan' \\ Jie $\mathrm{Fu}^{\mathrm{l}}$ \\ XiaoLing $\mathrm{Li}^{2}$ \\ AiPing Tong ${ }^{2}$ \\ YueLong Wang ${ }^{2}$ \\ YangMei Chen' \\ Gang Guo ${ }^{2}$ \\ 'Department of Neurology, The \\ Second Affiliated Hospital of \\ Chongqing Medical University, \\ Chongqing, ${ }^{2}$ State Key Laboratory of \\ Biotherapy and Cancer Center, and \\ Department of Neurosurgery, West \\ China Hospital, Sichuan University, \\ and Collaborative Innovation Center \\ for Biotherapy, Chengdu, People's \\ Republic of China
}

\begin{abstract}
Spinal cord injury (SCI) commonly leads to lifelong disability due to the limited regenerative capacity of the adult central nervous system. Nanomicelles can be used as therapeutic systems to provide effective treatments for SCI. In this study, a novel triblock monomethyl poly(ethylene glycol)-poly(L-lactide)-poly(trimethylene carbonate) copolymer was successfully synthesized. Next, polymeric nanomicelles loaded with zonisamide (ZNS), a Food and Drug Administration-approved antiepileptic drug, were prepared and characterized. The ZNS-loaded micelles (ZNS-M) were further utilized for the treatment of SCI in vitro and in vivo. The obtained ZNS-M were $\sim 50 \mathrm{~nm}$ in diameter with good solubility and dispersibility. Additionally, these controlled-release micelles showed significant antioxidative and neuron-protective effects in vitro. Finally, our results indicated that ZNS-M treatment could promote motor function recovery and could increase neuron and axon density in a hemisection SCI model. In summary, these results may provide an experimental basis for the use of ZNS-M as a clinically applicable therapeutic drug for the treatment of SCI in the future.
\end{abstract}

Keywords: spinal cord injury, zonisamide, nanomicelles

\section{Introduction}

Spinal cord injury (SCI) can result in devastating lifelong disability for patients and their families. In developing countries, the incidence rates of SCI have ranged from 12.7 to 29.7 cases per million people in the past few decades. ${ }^{1}$ According to a largescale epidemiological study of spinal injuries in mainland China, SCI accounted for $0.74 \%$ of all traumas from 2001 to $2007 .{ }^{2}$ Currently, medical treatment options after a spinal cord trauma are limited. The drug used clinically to treat acute SCI is an extremely large dose of methylprednisolone (MP) administered within the first $8 \mathrm{~h}$ post-injury. ${ }^{3}$ However, the efficacy of MP treatment is highly controversial due to the serious side effects associated with this glucocorticoid, including myopathy, infections and gastric bleeding. ${ }^{4,5}$ Moreover, there is no successful treatment for any of the main types of dysfunction that occur after SCI, including chronic severe pain, autonomic dysreflexia and bladder, bowel and sexual dysfunction. ${ }^{3}$ Therefore, the development of novel therapeutic strategies that target this condition is imperative.

The pathophysiology of SCI consists of a primary injury along with sequential secondary damages emanating from a multicascade of pathological mechanisms, which often leads to permanent motor and sensory deficits due to the limited regenerative capacity of the adult central nervous system (CNS). ${ }^{6}$ Oxidative stress and reactive oxygen species (ROS) production play a predominant role in the secondary injury mechanisms of SCI. ${ }^{7}$ Oxidative damage most likely contributes to calpain activation 
by the disruption of $\mathrm{Ca}^{2+}$ homeostasis. It is also known to exacerbate excitotoxic glutamate and to compromise the neuronal homeostatic mechanisms that either directly or indirectly regulate intracellular $\mathrm{Ca}^{2+}$, including $\mathrm{Ca}^{2+}$ ATPase, $\mathrm{Na}^{+}-\mathrm{K}^{+}$ATPase, $\mathrm{Na}^{+}-\mathrm{Ca}^{2+}$ exchanger and mitochondrial $\mathrm{Ca}^{2+}$ sequestration. ${ }^{8,9}$ Therefore, therapeutically targeting oxidative enzymes will prevent axonal demyelination, preserve oligodendrocyte integrity and spare neuronal loss. ${ }^{10}$

Zonisamide (1,2-benzisoxazole-3-methanesulfonamide; ZNS) is an antiepileptic drug that has already been approved for clinical use. Several clinical studies have reported the wide clinical spectrum of ZNS against both psychiatric and neurological disorders. The major antiepileptic action mechanisms of ZNS include inhibition of the voltage-gated $\mathrm{Na}^{+}$channel, the T-type voltage-sensitive $\mathrm{Ca}^{2+}$ channel, the $\mathrm{Ca}^{2+}$-releasing system and neuronal depolarization-induced glutamate release. ${ }^{11}$ Another previous study has shown that ZNS is an effective inhibitor of monoamine oxidase $\mathrm{B}$ (MAO-B) in vitro and in vivo. ${ }^{12}$ There is also information available showing that ZNS can induce protective effects against oxidative stress and the antioxidant redox system through modulation of cytosolic $\mathrm{Ca}^{2+}$ concentrations in neuronal cell lines. ${ }^{13}$ In addition, it has been proven that ZNS improves clinical outcome when used as an adjuvant in the treatment of Parkinson's disease (PD), possibly due to its ability to promote the recovery of degenerating dopaminergic neurons. ${ }^{11}$

Micelles are formed by the self-assembly of amphiphilic block copolymers in aqueous environment, and they can function as solubilizing agents for hydrophobic drugs. ${ }^{14,15}$ The hydrophobic segments of micelles become packed together in aggregates (core), which serve as a storage site for poorly water-soluble drugs and can act as a nanodepot for these agents. ${ }^{16,17}$ The common advantage of these drug delivery systems is that micelles are resistant to glomerular filtration, which could extend their retention time in the blood. ${ }^{18}$ Furthermore, micelles can facilitate the passage of drugs through the blood-brain barrier and blood-spinal cord barrier (BSCB), increasing therapeutic efficacy and safety compared to conventional pharmaceutical dosage forms. ${ }^{19}$ There have been several reports of biodegradable polymeric micelles for SCI treatment. ${ }^{20}$ Chen et $\mathrm{al}^{21}$ improved the bioavailability of MP in the spinal cord using poly(ethylene oxide)-poly(propylene oxide)-poly(ethylene oxide) (PEO-PPO-PEO) polymeric micelles as a delivery vehicle. In addition, self-assembled monomethoxy poly(ethylene glycol)-poly(D,L-lactic acid) di-block copolymer micelles (MPEG-PDLLA) have been demonstrated to be effective in restoring compound action potentials (CAPs) of the injured spinal cord, decreasing calcium influx into axons and promoting the recovery of locomotion function in an SCI rat model. ${ }^{22}$

Polyethylene glycol (PEG) is a fusogen that can restore the integrity of severed cell membranes. ${ }^{22,23}$ This water-soluble polymer is an ideal candidate for neural restoration in the clinic. PEG-based nanoparticles, including PEG-polyester, monomethoxy PEG-poly(D,L-lactic acid), PEG-silica and poly(lactic-co-glycolic acid), were demonstrated to act in CNS neuroprotection by repairing damaged axonal membranes through an intrinsic antioxidant mechanism. ${ }^{22,24-27}$ Poly(trimethylene carbonate) (PTMC) is a highly elastic polymer that was investigated for potential biomedical applications in soft tissue engineering and drug delivery systems. ${ }^{28}$ Block copolymers and terpolymers with polycarbonate segments have become promising bioresorbable materials. By changing the ratio and the total number of segments of the copolymers, the structure of micelles and their composition can be controlled. ${ }^{29,30}$ In addition, the mechanical properties, the hydrophilic-hydrophobic balance and the biodegradation rate of the materials can be easily modified by changing the block lengths and the composition of the polymers. ${ }^{31}$

In this study, we first synthesized a monomethyl poly(ethylene glycol)-poly(L-lactide)-poly(trimethylene carbonate) (MPEG-PLLA-PTMC) triblock copolymer. Furthermore, ZNS-loaded MPEG-PLLA-PTMC nanomicelles (ZNS-M) were successfully prepared. Finally, the monodisperse polymeric micelles were characterized by particle size distribution, transmission electron microscope (TEM), X-ray diffraction (XRD) and Fourier transform infrared (FTIR) spectroscopy, among others, and its neuroprotective effect was investigated in vitro and in vivo.

\section{Materials and methods \\ Materials, cell lines and animals}

ZNS was purchased from Aike Chemicals (Ningbo, China). Monomethyl poly(ethylene glycol) (MPEG; $M_{\mathrm{n}}=1.98 \times 10^{3}$; Fluka, Sigma-Aldrich Co., St Louis, MO, USA), L-lactic acid (L-LA; Sigma-Aldrich, USA), 1,3-trimethylene carbonate (TMC; Huizhou Foryou Medical Devices, China) and stannous octoate ( $\mathrm{Sn}(\mathrm{Oct})_{2}$; Sigma-Aldrich Co., St Louis, MO, USA) were used without purification. Methanol (high-performance liquid chromatography [HPLC] grade; Thermo Fisher Scientific, Waltham, MA, USA), thiazolyl blue tetrazolium bromide (MTT; Sigma), Cy3 Goat anti-Rabbit kit (Boster, Wuhan, Hubei, China) and FITC Goat anti-Mouse kit (Boster) were used as received. All the organic solvents used in this study were of analytical reagent (AR) grade and were used as received. 
The astrocyte rat CTX TNA2 (ATCC ${ }^{\circledR}$ CRL-2006 ${ }^{\mathrm{TM}}$ ) cell lines were cultured in Dulbecco's Modified Eagle's Medium (DMEM; HyClone, Logan, UT, USA) with 10\% fetal bovine serum (FBS; Gibco, Mulgrave Victoria, Australia) and 1\% penicillin/streptomycin (Gibco). The cells were incubated at $37^{\circ} \mathrm{C}$ with a humidified $5 \% \mathrm{CO}_{2}$ atmosphere.

Female Sprague-Dawley rats (HFK Bioscience Co., LED, Beijing, China) weighing $250 \pm 20 \mathrm{~g}$ were used as spinal cord hemisection models to evaluate neuroprotective effects and functional recovery in vivo. All mice were quarantined for a week before treatment and provided with standard laboratory animal living conditions. All animal procedures in this study were performed following protocols approved by the Institutional Animal Care and Treatment Committee of Sichuan University (Chengdu, People's Republic of China).

\section{Synthesis of the MPEG-PLLA-PTMC copolymer}

The copolymer with an MPEG-PLLA-PTMC ratio of 2000 $1000-1000$ was synthesized by ring-opening polymerization of L-lactide and TMC initiated by MPEG. Briefly, MPEG $(10 \mathrm{~g})$, L-lactide $(5 \mathrm{~g})$ and TMC $(5 \mathrm{~g})$ were introduced into the reaction vessel under a dry nitrogen atmosphere. The reaction was catalyzed by $\mathrm{Sn}(\mathrm{Oct})_{2}$ and kept at $130^{\circ} \mathrm{C}$ for $24 \mathrm{~h}$. The purified MPEG-PLLA-PTMC copolymer was kept in a desiccator and characterized by ${ }^{1} \mathrm{H}-\mathrm{NMR}$ (Varian 400 spectrometer; Varian Medical Systems, Palo Alto, CA, USA). In addition, the MPEG-PLLA-PTMC copolymer samples were dissolved in tetrahydrofuran (THF) to determine the molecular weights and distribution by GPC (1100 series; Agilent, Santa Clara, CA, USA).

\section{Preparation and characterization of the ZNS micelles (ZNS-M)}

ZNS-M were obtained through a single-step solid dispersion method. Briefly, ZNS and MPEG-PLLA-PTMC copolymer were dissolved in $25 \mathrm{~mL}$ acetone under mild stirring at room temperature. Then, the mixed solution was evaporated using a rotary evaporator at $37^{\circ} \mathrm{C}$ under gradually increasing vacuum. The resulting residue was dissolved in $4 \mathrm{~mL}$ normal saline (NS) at $37^{\circ} \mathrm{C}$ to form ZNS-loaded micelles. The obtained ZNS-M were filtered through a $0.22 \mathrm{~mm}$ syringe filter (Millex-LG; EMD Millipore, Billerica, MA, USA) and frozen by liquid nitrogen to avoid collisions before lyophilizing. The lyophilized powder was kept at $4^{\circ} \mathrm{C}$ before further use.

The stability of ZNS-M in aqueous solution was evaluated qualitatively at room temperature over $48 \mathrm{~h}$. Precipitation implied the instability of ZNS-M. In addition, the particle size distribution of ZNS-M was determined within 30 min by dynamic light scattering (DLS) using a Malvern Nano-ZS 90 laser particle size analyzer at $25^{\circ} \mathrm{C}$. All reported experimental results were performed in triplicate as mean \pm standard deviation (SD).

The morphological characteristics of ZNS-M were investigated by TEM (Tecnai G2 F20 S-TWIN electron microscope, FEI Co.) as previously described. ${ }^{23}$

Drug loading (DL) and encapsulation efficiency (EE) of ZNS-M were estimated by HPLC (Waters Alliance 2695). Briefly, $10 \mathrm{mg}$ of lyophilized ZNS-M powder was dissolved in $0.1 \mathrm{~mL}$ of methanol. The samples were analyzed in the mobile phase, containing 65\% (v/v) methanol and 35\% (v/v) ultrapure water, at a flow rate of $1 \mathrm{~mL} / \mathrm{min}$. Detection was performed on a Waters 2996 detector. Chromatographic separations were performed on a reversed-phase $\mathrm{C} 18$ column $\left(4.6 \times 250 \mathrm{~mm}-5 \mu \mathrm{m}\right.$, Grace Analysis column) at $28^{\circ} \mathrm{C}$. The detection wavelength for ZNS was set at $240 \mathrm{~nm}$, and the elution time of ZNS was 2.218 min under the chromatographic conditions described earlier. The concentration of ZNS in the solution was determined by HPLC and calculated using the following equations:

$$
\begin{gathered}
\% \mathrm{DL}=\frac{\text { Drug }}{\text { Polymer }+ \text { Drug }} \times 100 \\
\% \mathrm{EE}=\frac{\text { Experimental drug loading }}{\text { Theoretical drug loading }} \times 100
\end{gathered}
$$

XRD (X Per Pro MPD DY 1291; PHILIPS, Amsterdam, the Netherlands) measurements were performed to study the phase and crystallinity of the ZNS-M powder at a scanning rate of and a step size of $0.03^{\circ}$ in the $2 \theta$ range from $5^{\circ}$ to $50^{\circ}$.

The chemical structure of the ZNS-M powder was determined by FTIR spectroscopy (NICOLET 200SXV; Nicolet, New York, NY, USA). Infrared spectra were recorded in a wavenumber range of $400-4,000 \mathrm{~cm}^{-1}$.

\section{In vitro release study}

The release kinetics of ZNS from ZNS-M were determined by a dialysis method. A solution of ZNS-M and free ZNS in dimethyl sulfoxide (DMSO) $(0.5 \mathrm{~mL}, 0.5 \mathrm{mg} / \mathrm{mL})$ were placed into dialysis bags (molecular weight cutoff, $3.5 \mathrm{kDa}$ ). The dialysis bags were incubated in $30 \mathrm{~mL}$ of phosphate buffer solution (PBS, pH 7.4) containing Tween-80 (0.5 wt\%) at $37^{\circ} \mathrm{C}$ with gentle shaking $(100 \mathrm{rpm})$. At predetermined 
time points, $1 \mathrm{~mL}$ of the medium was collected and stored at $-20^{\circ} \mathrm{C}$ for further analysis. The remaining medium was removed and replaced by prewarmed fresh PBS. The collected samples were quantified using HPLC. All results are presented as the mean of five sample runs, expressed as the mean $\pm \mathrm{SD}$.

\section{In vitro antioxidant activity and cytotoxicity test}

The CTX TNA2 cells were seeded at $1 \times 10^{3}$ cells per well into a 96-well plate and incubated for $12 \mathrm{~h}$ at $37^{\circ} \mathrm{C}$ in a humidified atmosphere containing $5 \% \mathrm{CO}_{2}$. After replacing with fresh medium, the cells were exposed to $500 \mu \mathrm{M}$ $\mathrm{H}_{2} \mathrm{O}_{2}$ solution and were treated with free ZNS (dissolved in DMSO), ZNS-M and blank micelles (M) at different concentrations $(0-50 \mu \mathrm{M})$. For the cytotoxicity test, $\mathrm{H}_{2} \mathrm{O}_{2}-$ untreated cells were incubated with free ZNS, ZNS-M and blank $\mathrm{M}$ at different concentrations $(0-50 \mu \mathrm{M})$. After the treatment, the cell viability was estimated with the MTT assay at particular time intervals according to previously published protocol. ${ }^{23}$

\section{Primary cortical neuron culture}

The cortical neuron cells were separated from embryonic SD mice. Briefly, after anesthesia, the cerebral cortexes of the embryonic mice were carefully dissected and washed with PBS (pH 7.4) on ice. The specimens were dissociated by $1 \mathrm{~mL}$ pipette and digested with $1 \mathrm{mg} / \mathrm{mL}$ collagenase II (Sigma-Aldrich) for $15 \mathrm{~min}$ at $37^{\circ} \mathrm{C}$ in an incubator. The collagenase was inactivated by adding DMEM with $10 \% \mathrm{FBS}$. After centrifugation, the supernatant was discarded and the pellet was resuspended in Neurobasal ${ }^{\circledR}$-A Medium with $2 \%$ B-27 ${ }^{\circledR}$ Serum-Free Supplement (Thermo Fisher Scientific, Waltham, MA, USA). The resulting solution was incubated in a $75 \mathrm{~cm}^{2}$ culture dish at $37^{\circ} \mathrm{C}$ with $5 \%$ carbon dioxide. The culture medium was changed every 3 days. Free ZNS, ZNS-M and blank $M$ were added into the culture medium at a final concentration of $20 \mu \mathrm{M}$ within $24 \mathrm{~h}$.

After 2 weeks, the neuronal cells were fixed with prewarmed 4\% paraformaldehyde solution (Boster) and blocked in $5 \%$ goat serum with $0.1 \%$ Triton-X (Sigma). After $30 \mathrm{~min}$, the cells were immunostained by the anti-MAP2 (1:1,000 dilution; Abcam, Cambridge, UK) and the anti-neurofilament-H (1:200 dilution; CST, Boston, USA) antibodies. The neuron cells and neurite lengths were observed under microscope. Quantification of the axon length and the average branch points was performed by counting the numbers randomly under high-power visual fields.
Surgical protocol and drug administration

Surgeries were performed after anesthetizing the SD rats with chloral hydrate solution (10 wt\%, $0.3 \mathrm{~mL} / 100 \mathrm{~g}$; Kelong, Chengdu, China). A $2 \mathrm{~cm}$ longitudinal skin incision was centered over the T9-T10 spinous process along the midline. Without disrupting the dura mater, the T10 spinal segment was exposed by removing the dorsal part of the vertebra. Next, the exposed cord was hemi-cut on the right side by a sharp blade. Finally, the muscle and skin layers were sutured.

After 24 h, $\sim 96 \%$ of the injured rats had survived. The animals were randomly divided into four main experimental groups (seven rats per group) receiving different treatments. The SCI rats were treated intravenously with ZNS-M (10 mg/ $\mathrm{kg}$ ZNS), free ZNS (10 mg/kg ZNS), equivalent blank M and NS both $6 \mathrm{~h}$ and 4 days after surgery. Before the injection, the lyophilized powder was redissolved in NS with $1 \mathrm{mg} /$ $\mathrm{mL}$ ZNS at $37.5^{\circ} \mathrm{C}$.

\section{Magnetic resonance imaging (MRI)}

After anesthesia, each rat was placed on the fixation system in prone position. The experiments were performed on a 3.0 T clinical MR scanner (Siemens Trio Tim), using a rat phased array RF coil (CG-MUC18-H300-AS; Chengguang Medical Technologies Co., Ltd, Shanghai, China). The spine MRIs were scheduled at 4 weeks post-SCI. Images of the injured regions were acquired in the sagittal planes. The sequence protocol included T1-weighted, 256×256 matrix, field of view (FOV) 80, slice thickness $1 \mathrm{~mm}$, echo time (TE) 13/92 ms and repetition time (TR) 505/3,000 ms, respectively.

\section{Functional analysis}

Functional recovery of the hind limbs was evaluated using the Basso-Beattie-Bresnahan locomotor rating scale (BBB score) ${ }^{32}$ at 1 week after treatment and subsequently every 2 weeks. The evaluations were performed using a double-blind method, and the average scores in each group were calculated.

\section{Immunohistochemical examination}

Specific proteins were identified using immunohistochemical staining as described in our previous study. Briefly, the samples of SCI were dissected and post-fixed in 4\% paraformaldehyde over $24 \mathrm{~h}$. After being blocked by 5\% goat serum, the coronal sections $(4 \mu \mathrm{m})$ were stained with the anti-glial fibrillary acidic protein (GFAP) antibody (1:5,000 dilution; Abcam) and the anti-neurofilament-H antibody (1:200 dilution; CST). After incubation with the primary antibody at $4^{\circ} \mathrm{C}$ for $12 \mathrm{~h}$, the sections were incubated with 
the fluorescent secondary antibody kits (Cy3 Goat anti-Rabbit and FITC Goat anti-Mouse) according to the manufacturer's instructions. Nuclei were counterstained for $3 \mathrm{~min}$ using DAPI. All samples were examined with a fluorescence microscope (Leica DM IRE2; Leica Microsystems, Wetzlar, Germany). The heart, liver, spleen, lung and kidneys were also excised and stained with hematoxylin-eosin (H\&E). Quantification of neurofilament and GFAP was performed by counting the number of positive cells in 15 high-power visual fields randomly. More than five sections of each sample were considered.

\section{Statistical analysis}

Statistical analysis was carried out using SPSS 15.0 software (Chicago, IL, USA). Comparisons of experimental data in different groups were performed using one-way analysis of variance (ANOVA). Results with $P<0.05$ were considered statistically significant.

\section{Results}

\section{Synthesis of the MPEG-PLLA-PTMC copolymer}

The biodegradable MPEG-PLLA-PTMC copolymer was successfully synthesized by the ring-opening polymerization of
L-LA and TMC initiated by MPEG (Figure 1). The numberaverage molecular weights $\left(M_{\mathrm{n}}\right)$ of the MPEG-PLLA-PTMC copolymers were calculated by ${ }^{1} \mathrm{H}-\mathrm{NMR}$ (Figure $2 \mathrm{~A}$ ). GPC results (Figure $2 \mathrm{~B}$ ) show that only one single peak was present. The $M_{\mathrm{n}}$ and $M_{\mathrm{w}}$ of MPEG-PLLA-PTMC were $4.40 \times 10^{3}$ and $5.97 \times 10^{3}$, respectively. The polydispersity index (PDI) of the copolymer was 1.5 .

\section{Preparation and characterization of ZNS-M}

The ZNS-loaded MPEG-PLLA-PTMC nanomicelles were prepared by a one-step solid dispersion method and were characterized in detail. As illustrated in Figure 3, blank M $(10 \mathrm{mg} / \mathrm{mL})$ and ZNS-M (10 mg/mL) were stable in NS with good solubility at room temperature. However, the solubility of free ZNS $(10 \mathrm{mg} / \mathrm{mL})$ in water was poor (Figure $3 \mathrm{C}$ ). The particle size of ZNS-M was $42.7 \pm 3.2 \mathrm{~nm}$ (Figure 3B) with a PDI of $0.167 \pm 0.026$. Furthermore, the properties of different mass ratios of ZNS-M (3 wt $\%, 5 \mathrm{wt} \%, 8 \mathrm{wt} \%$, $10 \mathrm{wt} \%$ and $13 \mathrm{wt} \%$ ) were evaluated. The results showed a reasonable increase in DL with a decrease in EE and stability time (Table 1). According to the TEM results, ZNS-M $(5 \mathrm{wt} \%)$ were well-dispersed spheres with a mean diameter of $\sim 50 \mathrm{~nm}$.

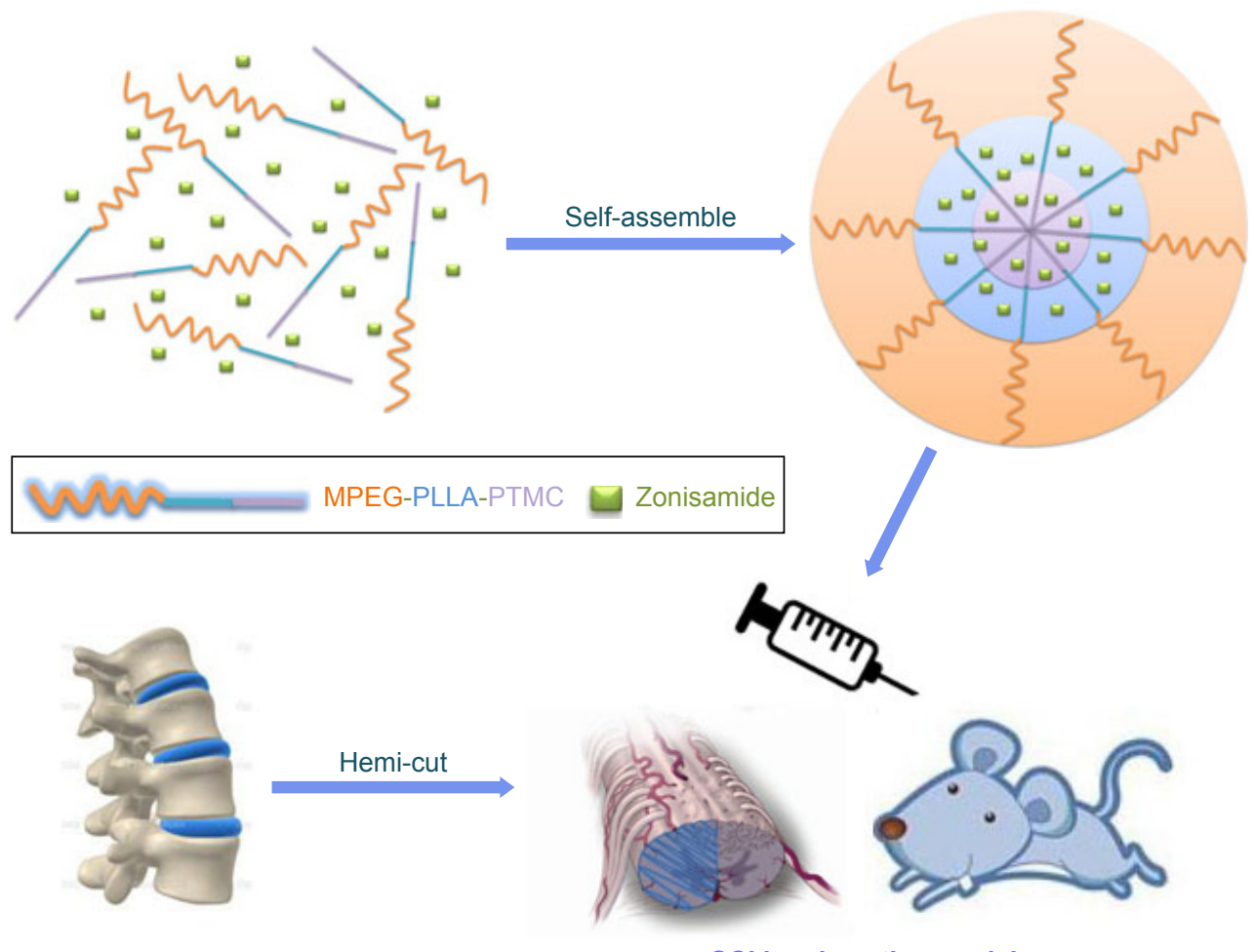

SCI hemi-section model

Figure I Preparation schemes of the ZNS-M synthesized by self-assembled assay and the drug administration process. Abbreviations: MPEG-PLLA-PTMC, monomethyl poly(ethylene glycol)-poly(L-lactide)-poly(trimethylene carbonate); SCI, spinal cord injury; ZNS-M, zonisamide micelles. 


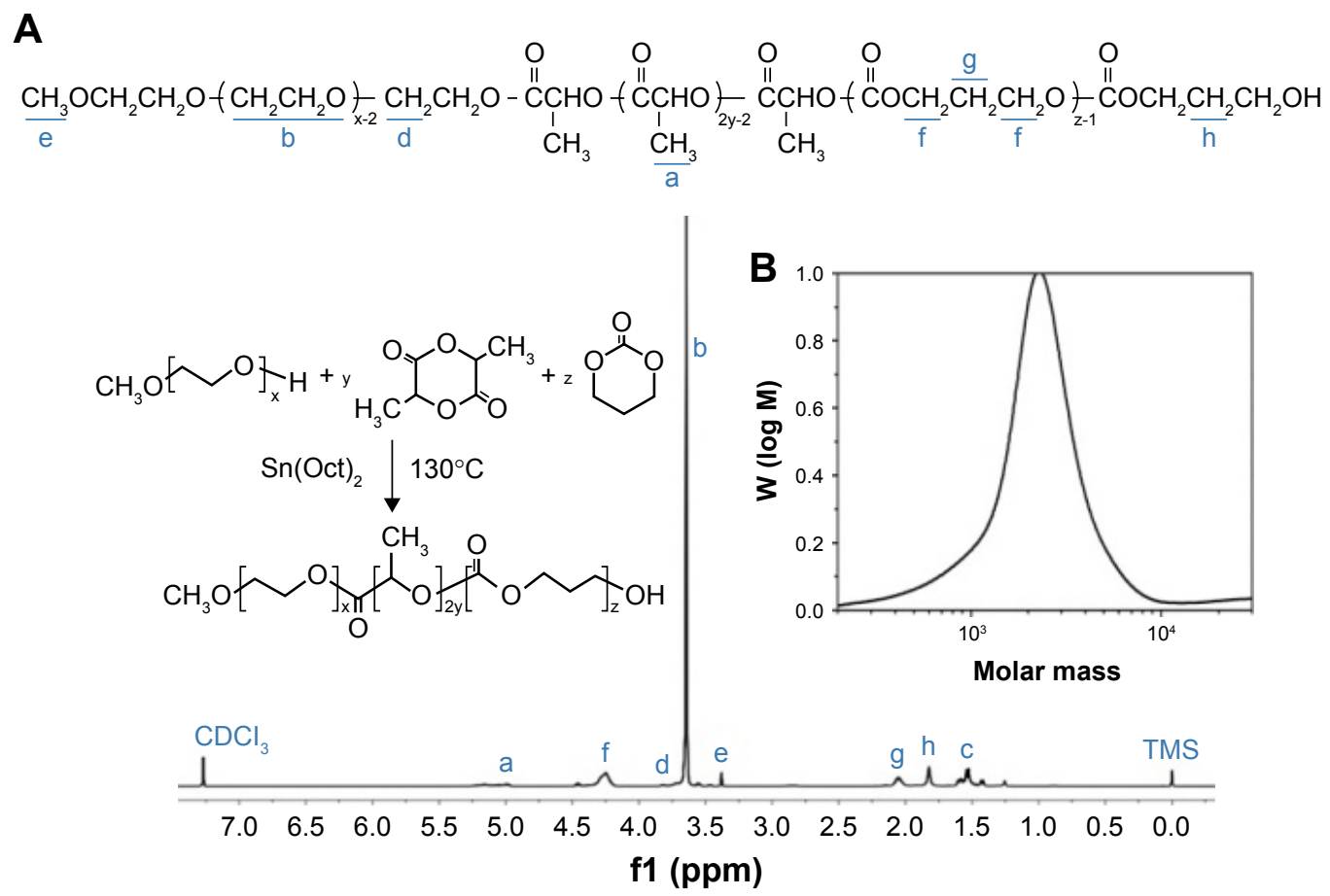

Figure 2 ' $\mathrm{H}-\mathrm{NMR}$ and GPC analysis.

Notes: (A) 'H-NMR and (B) GPC spectra of MPEG-PLLA-PTMC.

Abbreviation: MPEG-PLLA-PTMC, monomethyl poly(ethylene glycol)-poly(L-lactide)-poly(trimethylene carbonate).

FTIR spectroscopy shows the main characteristic bands of ZNS and the MPEG-PLLA-PTMC copolymer. In the spectrum of ZNS-M, the peaks at $1,605 \mathrm{~cm}^{-1}$ and $749.2 \mathrm{~cm}^{-1}$, which were not detected in the blank MPEG-PLLA-PTMC micelles, were shifted lower in comparison to free ZNS (Figure 4A). Moreover, the XRD spectra of free ZNS, blank $M$ and the ZNS-M crystals are shown in Figure 4B. The specific diffraction peaks of ZNS disappeared in the XRD spectrum of ZNS-M, indicating that ZNS was incorporated in the MPEG-PLLA-PTMC micelles.

\section{In vitro drug release behavior}

The in vitro drug release behavior of ZNS-M was determined by HPLC. As shown in Figure 5, ZNS was sustainably released from ZNS-M over a 10-day extended period. On the other hand, free ZNS in DMSO showed faster release behavior than ZNS-M. In the first $8 \mathrm{~h}, \sim 30.32 \% \pm 4.57 \%$ of ZNS was released from ZNS-M, which was lower than the amount released in the free ZNS group $(80.08 \% \pm 3.29 \%, P<0.05)$. The cumulative release of ZNS-M was $\sim 75.167 \% \pm 3.27 \%$ over the period of 11 days, which was much lower than the cumulative release of the free ZNS group (95.997 \pm 2.31 , $P<0.05)$. Thus, the MPEG-PLLA-PTMC copolymer micelles could facilitate the sustained release of ZNS as a drug delivery system.

\section{Antioxidative effects and cytotoxicity evaluation}

To investigate the antioxidant activity of ZNS-M in vitro, the number of viable $\mathrm{H}_{2} \mathrm{O}_{2}$-treated CTX TNA2 cells was quantified by MTT assay. After drug treatment for $24 \mathrm{~h}$ and $48 \mathrm{~h}$, the absorbance of the cells in the ZNS-M group was significantly increased compared to the DMSO and the control group $(P<0.05)$ (Figure 6A and $\mathrm{B})$. The free ZNS group also showed a higher viable cell number than the DMSO and the control group at $24 \mathrm{~h}$ and $48 \mathrm{~h}$, respectively (Figure 6A and B). Thus, ZNS may provide a neuroprotective effect against oxidative stress in nervous tissue. For $\mathrm{H}_{2} \mathrm{O}_{2}$ untreated cells, no significant differences were observed in all groups within $24 \mathrm{~h}$ (Figure 6C). However, it was notable that the absorbance of the ZNS-M group was lower than that of the other four groups (Figure 6D) at $48 \mathrm{~h}$. These results indicated that ZNS-M can inhibit the growth of neuroglia cells in vitro.

\section{Axon elongation effect of ZNS-M in vitro}

The axon lengths of primary neuron cells were measured under high-power visual fields after treatment with the drugs. In the ZNS and ZNS-M groups, the axon lengths of neurons were obviously elongated (Figure 7A and B) compared to 

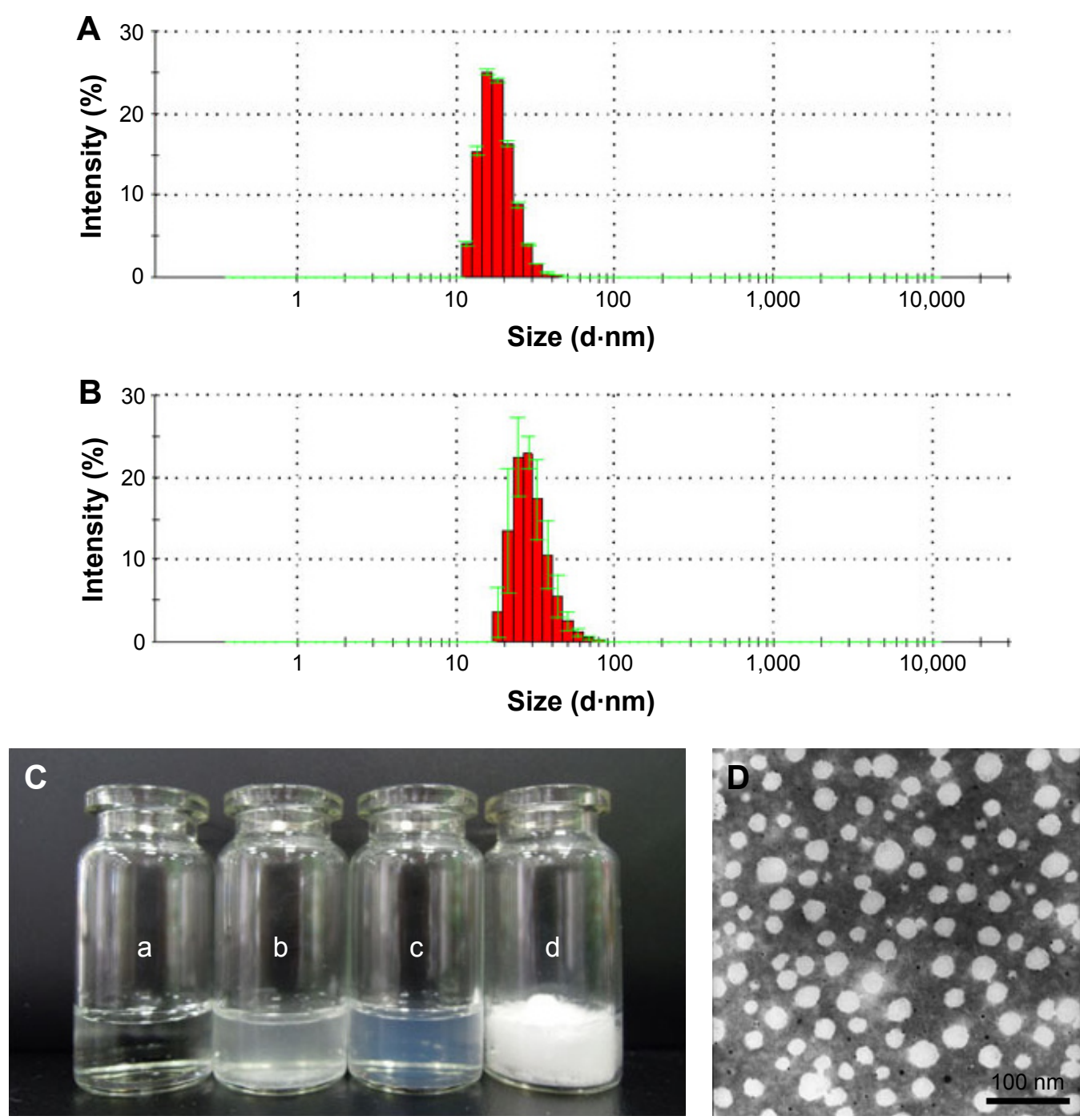

Figure 3 Characterization of ZNS-M.

Notes: Size distribution of (A) blank M and (B) ZNS-M; (C) photographs of different solutions: (a) water, (b) free ZNS in water, (c) ZNS-M and (d) lyophilized powder of ZNS-M; (D) TEM image of ZNS-M. Scale bar $=100 \mathrm{~nm}$.

Abbreviations: M, micelles; TEM, transmission electron microscope; ZNS, zonisamide; ZNS-M, zonisamide micelles.

those in the control group after 2 weeks. In addition, the number of branch points was also increased in the ZNS and ZNS-M groups (Figure 7A and C). However, there was no significant difference in either the axon lengths or the branch points between the ZNS group and the ZNS-M group

Table I Characterization of prepared ZNS-M

\begin{tabular}{llll}
\hline ZNS/MPEG-PLLA-PTMC & DL (\%) & EE (\%) & Stability (h) \\
\hline $3: 97$ & $2.83 \pm 0.03$ & $94.33 \pm 0.28$ & 38 \\
$5: 95$ & $4.56 \pm 0.06$ & $91.20 \pm 0.31$ & 12 \\
$8: 92$ & $7.62 \pm 0.05$ & $95.25 \pm 0.67$ & 3.5 \\
$10: 90$ & $7.84 \pm 0.03$ & $78.40 \pm 1.39$ & 1 \\
$13: 87$ & $10.79 \pm 0.04$ & $83.00 \pm 1.21$ & 0.15 \\
\hline
\end{tabular}

Abbreviations: MPEG-PLLA-PTMC, monomethyl poly(ethylene glycol)-poly(Llactide)-poly(trimethylene carbonate); DL, drug loading; EE, encapsulation efficiency; ZNS, zonisamide; ZNS-M, zonisamide micelles.
(Figure 7B and C). These results indicated the abilities of ZNS-M to enhance neurite elongation in primary neurons.

\section{$M R I$ and $B B B$ score analysis}

After 1 month, the pathological change of SCI was examined by a 3.0 T MRI unit. The different degrees of scarring tissue adhesion were observed in each group (Figure 8Aa-d). However, in the ZNS-M group (Figure 8Ac), the atrophied area of the spinal cord was obviously smaller than that in other SCI groups.

To further evaluate motor function in the SCI models, the locomotor rating scale was performed every 2 weeks. As shown in Figure 8B, the hind limbs of all experimental animals (except the sham group) were paralyzed after the injury. The BBB scores of the sham group were always at the highest level because the nerve function was not damaged. The mean 

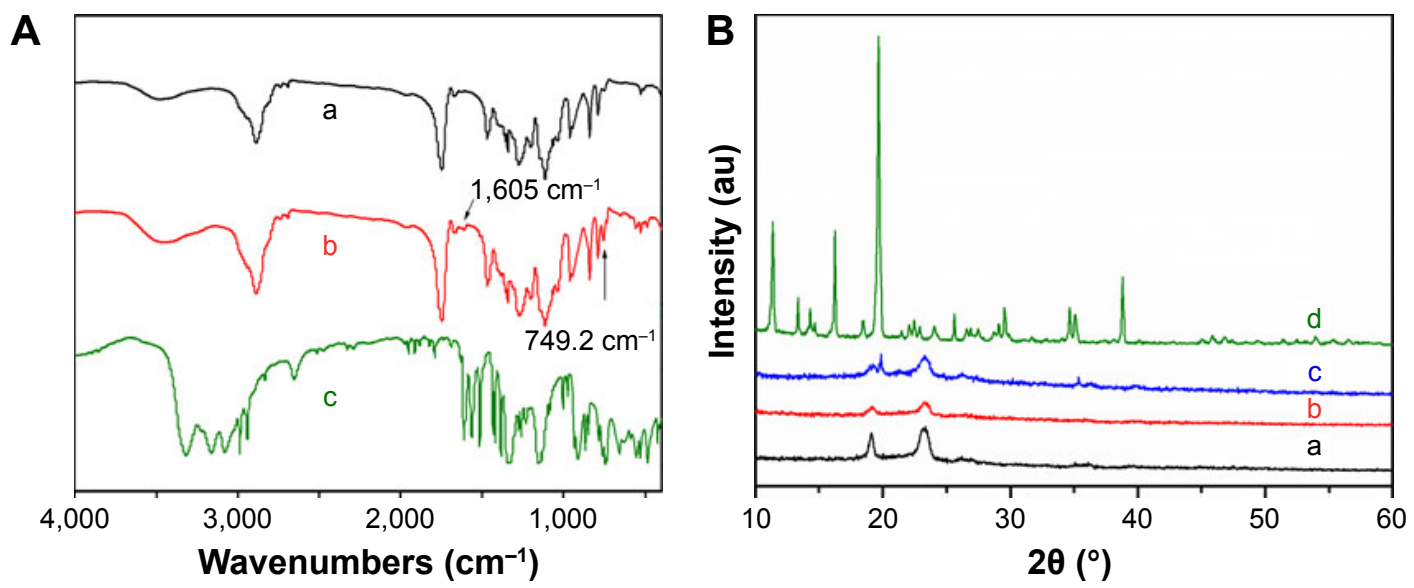

Figure 4 FTIR and XRD analysis.

Notes: (A) FTIR spectra of (a) ZNS-M, (b) blank M and (c) free ZNS. (B) XRD patterns of (a) ZNS-M, (b) blank M, (c) free ZNS and (d) free ZNS mixture with blank M powder.

Abbreviations: FTIR, Fourier transform infrared; XRD, X-ray diffraction; M, micelles; ZNS, zonisamide; ZNS-M, zonisamide micelles.

BBB scores of the control group were lower than those of the ZNS and ZNS-M groups at each time point. During the first 14 days, the average BBB scores of the ZNS group were slightly higher than the scores of the ZNS-M group. From the 14th day, the scores of the ZNS-M group continuously increased from 5.5 to 12 (21 days after injury), exceeding the scores of the free ZNS group. Notably, significant differences gradually emerged between each groups after 4 weeks postSCI $(P<0.05)$. These results suggested that ZNS-M plays a role in the improvement of neurological function.

\section{Histological observation and toxicity evaluation in vivo}

The histopathological manifestations in injured spinal tissues at 4 weeks post-operation were analyzed. Immunofluorescence

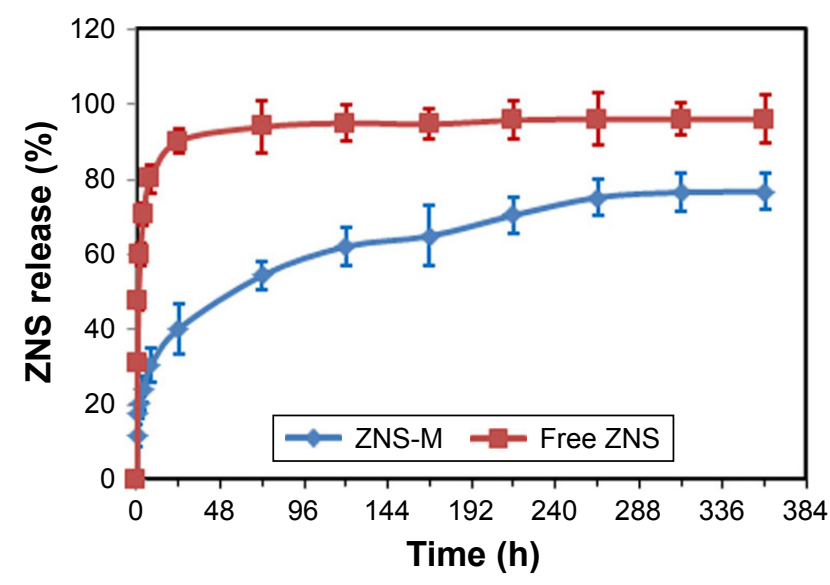

Figure 5 Drug release behavior of ZNS-M and free ZNS in vitro. Note: Error bars correspond to SD.

Abbreviations: SD, standard deviation; ZNS, zonisamide; ZNS-M, zonisamide micelles. staining showed that NF-positive nerve fibers were distributed in the middle areas of the spinal cord in the sham group (Figure 9A). The number of NF-positive nerve fibers in the ZNS-M group was significantly higher than in the free ZNS and control groups (Figure 9A). Additionally, in the area of the spinal injury, nerve fibers extended over a long distance (Figure 9A), indicating that ZNS-M treatment is conducive for nerve fiber repair in vivo. GFAP is an important marker located in glial cells. Immunofluorescence staining showed intense GFAP distribution at the injured site of the spinal cord in the ZNS-M; however, ZNS-M treatment did not result in the same significant reduction in the number of GFAPpositive neurons that it did in vitro (Figure 9B). In addition, the safety evaluation indicated that the injected ZNS-M, free ZNS and blank $\mathrm{M}$ at $10 \mathrm{mg} / \mathrm{kg}$ displayed no acute toxicity in vivo (Figure 10).

\section{Discussion}

Strategies for treating SCI can be broadly divided into two main types: neuroprotection and regeneration. The detrimental effects of oxidative stress commence immediately after the primary impact of SCI, causing membrane and cellular damage. ${ }^{33,34}$ Thus, antioxidative therapy is a reasonable approach to arrest and reverse inflammatory response in SCI. ZNS is a Food and Drug Administration-approved antiepileptic drug in clinical practice. It has been reported that ZNS is a MAO-B inhibitor that is effective in lessening neuron-specific oxidative stress. ${ }^{12}$ It has been proposed that the mechanisms of its neuroprotective effects include free radical scavenging and reducing oxidative stress. The predominant feature of SCI is damage to axon tracts, resulting 

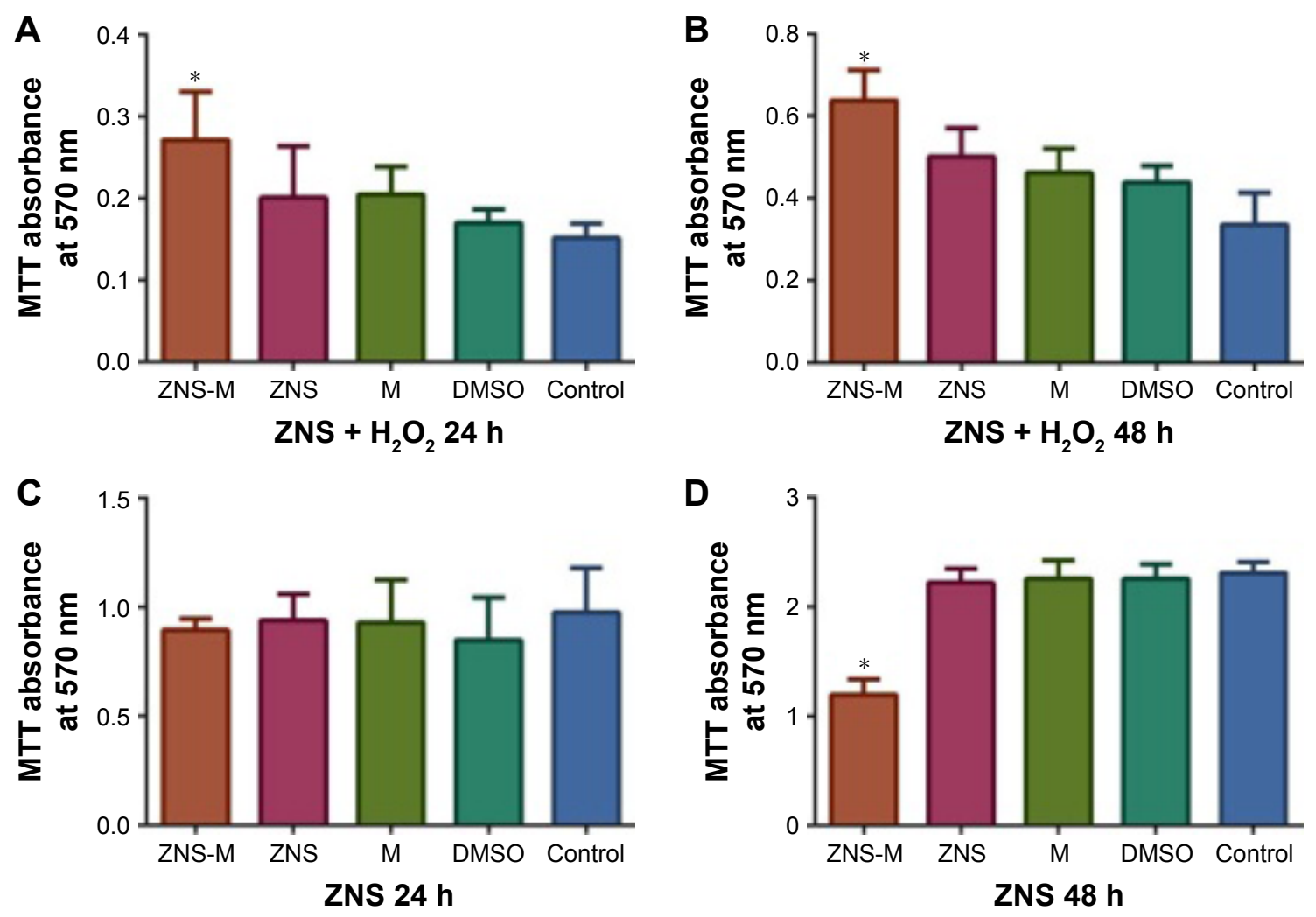

Figure 6 Antioxidative effects and cytotoxicity evaluation.

Notes: The absorbance of $\mathrm{H}_{2} \mathrm{O}_{2}$-exposed CTX TNA2 cells treated with ZNS-M, free ZNS and blank M at (A) $24 \mathrm{~h}$ and (B) $48 \mathrm{~h}$. The viabilities of CTX TNA2 cells treated with ZNS-M, free ZNS and blank M at (C) $24 \mathrm{~h}$ and (D) $48 \mathrm{~h} . * \mathrm{P}<0.05$.

Abbreviations: M, micelles; MTT, thiazolyl blue tetrazolium bromide; DMSO, dimethyl sulfoxide; ZNS, zonisamide; ZNS-M, zonisamide micelles.

in loss of communication between the CNS and the body. ${ }^{35,36}$ Thus, the challenge for spinal cord regeneration is not simply to fill in a lesion with a new cellular matrix but rather to recapitulate the highly organized axon pathways that were lost. ${ }^{37}$ In addition to reforming neural circuits, axon growth is an important component of regeneration. A recent study suggested that ZNS is able to enhance neurite elongation and neurite regeneration of primary motor neurons. ${ }^{38}$ Thus, we expect that the drug function mechanism of ZNS will play a role in the treatment of SCI.

Nanotechnology-based drug delivery has become an emerging strategy that provides particular advantages for neuroprotection. ${ }^{33}$ Micelles have been used as drug carriers for many years. Hydrophobic drugs can be encapsulated in the core to improve their water solubility, stability and circulation half-life. In previous studies, PEG has been commonly used as the hydrophilic moiety of micelles due to its solubility. ${ }^{23,39}$ The amphiphilic polymeric components (eg, MPEG-PDLLA micelles) are able to seal the damaged cell membranes and play a neuroprotective role. ${ }^{22}$ In this study, a novel MPEG-PLLA-PTMC triblock copolymer was successfully synthesized by ring-opening polymerization.
In subsequent studies, the ZNS-loaded MPEG-PLLA-PTMC micelles (ZNS-M) were successfully prepared by a one-step solid dispersion method without the use of any surfactants or toxic organic solvents. Detailed characterization of ZNS-M showed their favorable stability, along with their small particle sizes and high EE and DL (Table 1; Figure 3). The results of TEM further affirmed that ZNS-M were stable and monodisperse in aqueous solution (Figure 3D), which suggests that they could be used for intravenous applications. The main characteristic bands in the FTIR and XRD spectra indicated that ZNS was completely and amorphously encapsulated in the core-shell structure of the MPEG-PLLAPTMC micelles (Figure 4). The lasting release behavior of ZNS-M was observed in vitro (Figure 5). However, complete release was not seen in this drug release studies, which may be due to the drug aggregating over time in the release medium or during the freezing of the release samples before measurement. ${ }^{16,40,41}$

Inflammation plays a role in the progression of secondary SCI. The activated microglial cells can induce oxidative stress when cultured with neuronal cells. ${ }^{42,43}$ Although the response is natural, oxidative stress can be detrimental to 

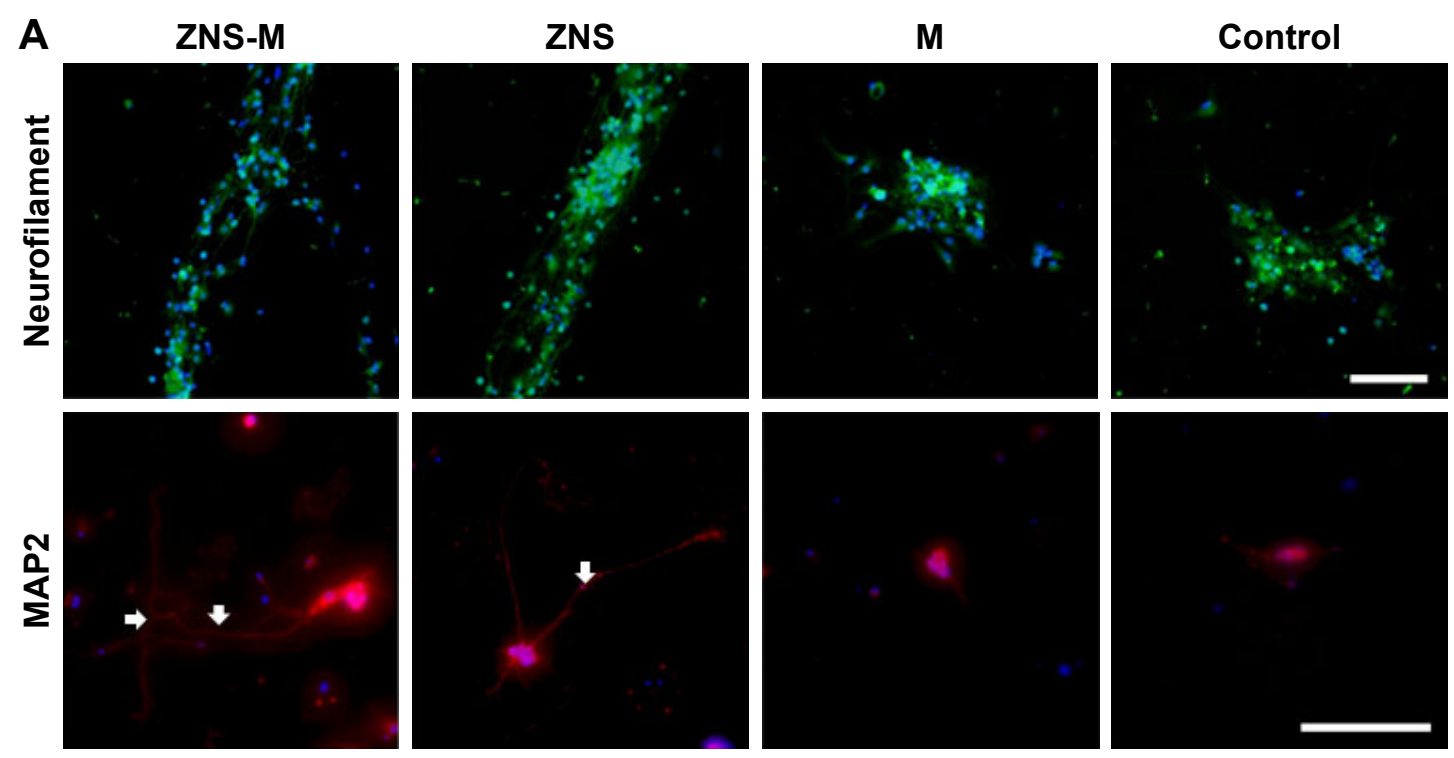

B

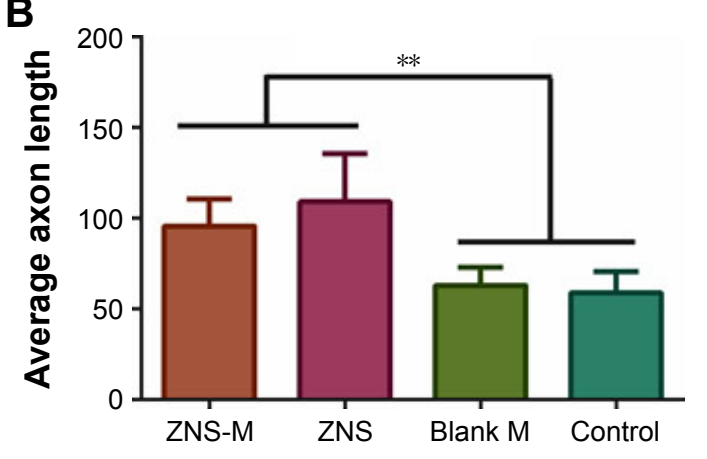

C

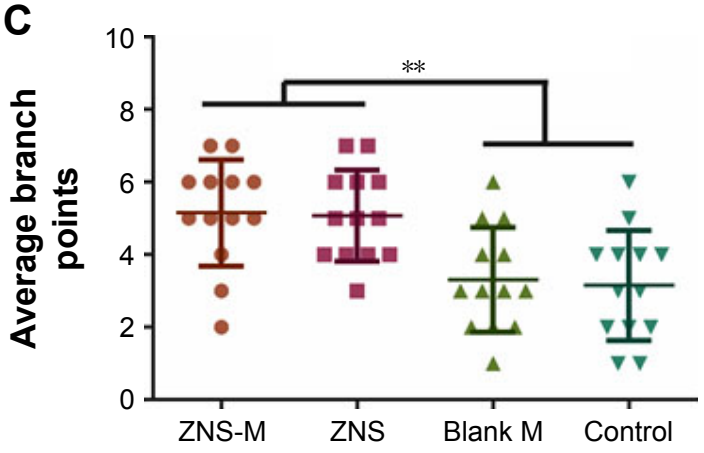

Figure 7 ZNS-M increases the number of branch points and neurite length in primary neuron cells.

Notes: (A) The images of branch points (neurofilament; green) and axon lengths (MAP2; red) of neurons. Arrows indicate the elongated axons. (B) The neurite lengths and (C) the number of branch points were significantly increased in the ZNS-M group and the free ZNS group. Quantitative data are represented as mean \pm SD. *P $<0.05$ was considered significant $(* * P<0.01)$. Scale bar $=100 \mu \mathrm{m}$.

Abbreviations: SD, standard deviation; M, micelles; ZNS, zonisamide; ZNS-M, zonisamide micelles.
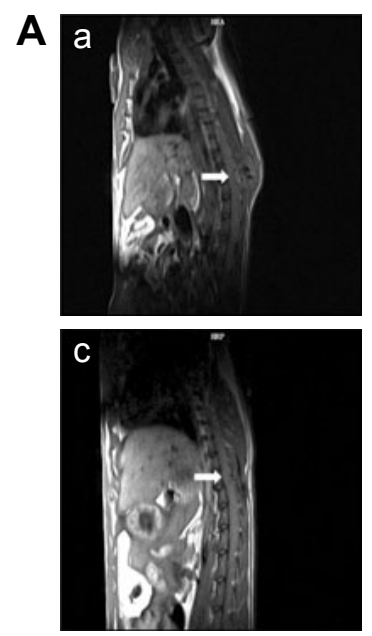
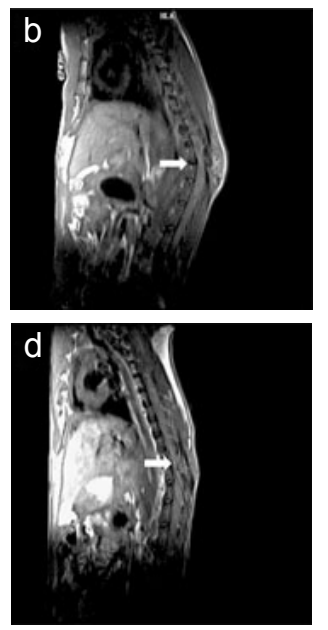

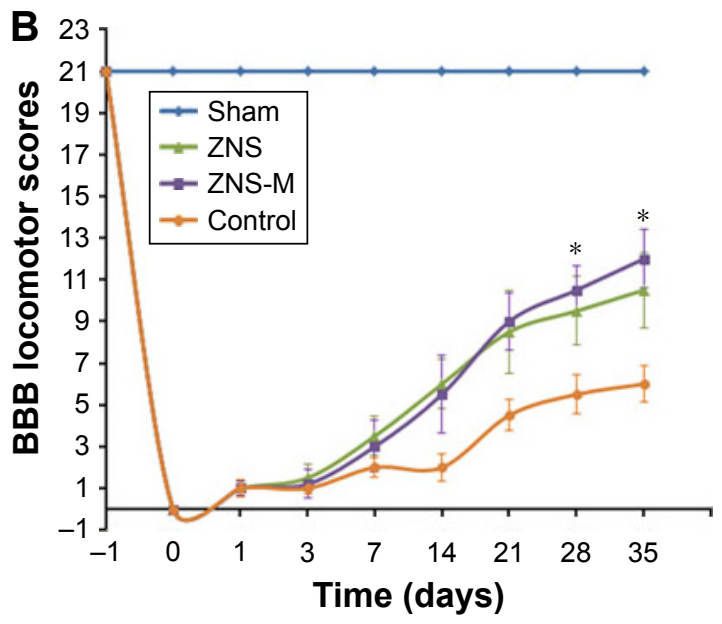

Figure 8 Functional analysis.

Notes: (A) MRI images of SCl rats after 4 weeks: (a) sham group, (b) free ZNS group, (c) ZNS-M group and (d) control. Arrows indicate the site of injury. (B) BBB scores of each treated group. $* P<0.05$.

Abbreviations: MRI, magnetic resonance imaging; SCI, spinal cord injury; BBB, Basso-Beattie-Bresnahan; ZNS, zonisamide; ZNS-M, zonisamide micelles. 

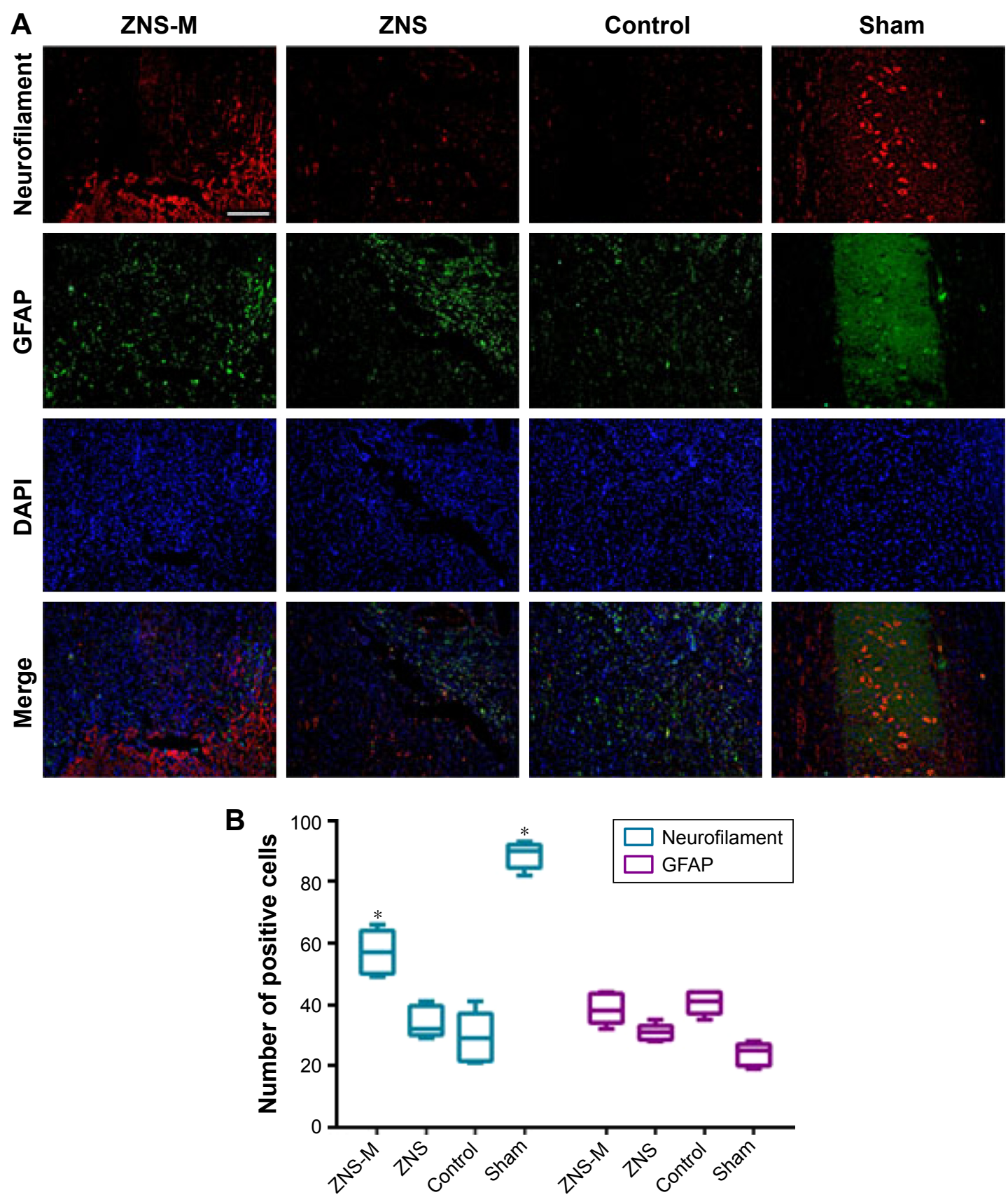

Figure 9 Histological analysis in $\mathrm{SCl}$ rats at 4 weeks.

Notes: (A) Representative immunohistologic images of neurofilament (red), GFAP (green) and DAPI (blue) in different groups. (B) Quantitative analyses of neurofilamentand GFAP-positive cells under high-power visual fields. Quantitative data are represented as mean \pm SD. $* P<0.05$ was considered significant. Scale bar $=100 \mu \mathrm{m}$. Abbreviations: SCl, spinal cord injury; GFAP, glial fibrillary acidic protein; SD, standard deviation; ZNS, zonisamide; ZNS-M, zonisamide micelles.

cells that are already undergoing stress. Herein, we identified that ZNS-M effectively inhibited $\mathrm{H}_{2} \mathrm{O}_{2}$-induced cell death in rat astrocyte cells. The results indicated that ZNS-M and free ZNS were protective against oxidative stress (Figure 6A and $\mathrm{B}$ ). The viable cell number in the ZNS-M group was significantly higher than other groups. This result may partially stem from the PEG segment, which has the ability to recover axonal integrity. ${ }^{19}$ In addition, ZNS-M exhibited an inhibitory effect on neuroglia cell growth, which may help inhibit glial scar formation. ${ }^{44}$ In addition to the antioxidant effects, it was also shown that ZNS has the ability to promote neurite elongation in mouse primary motor neurons in a dosedependent manner. ${ }^{38}$ In this study, we found that the axon lengths of neurons were obviously elongated in the ZNS and 

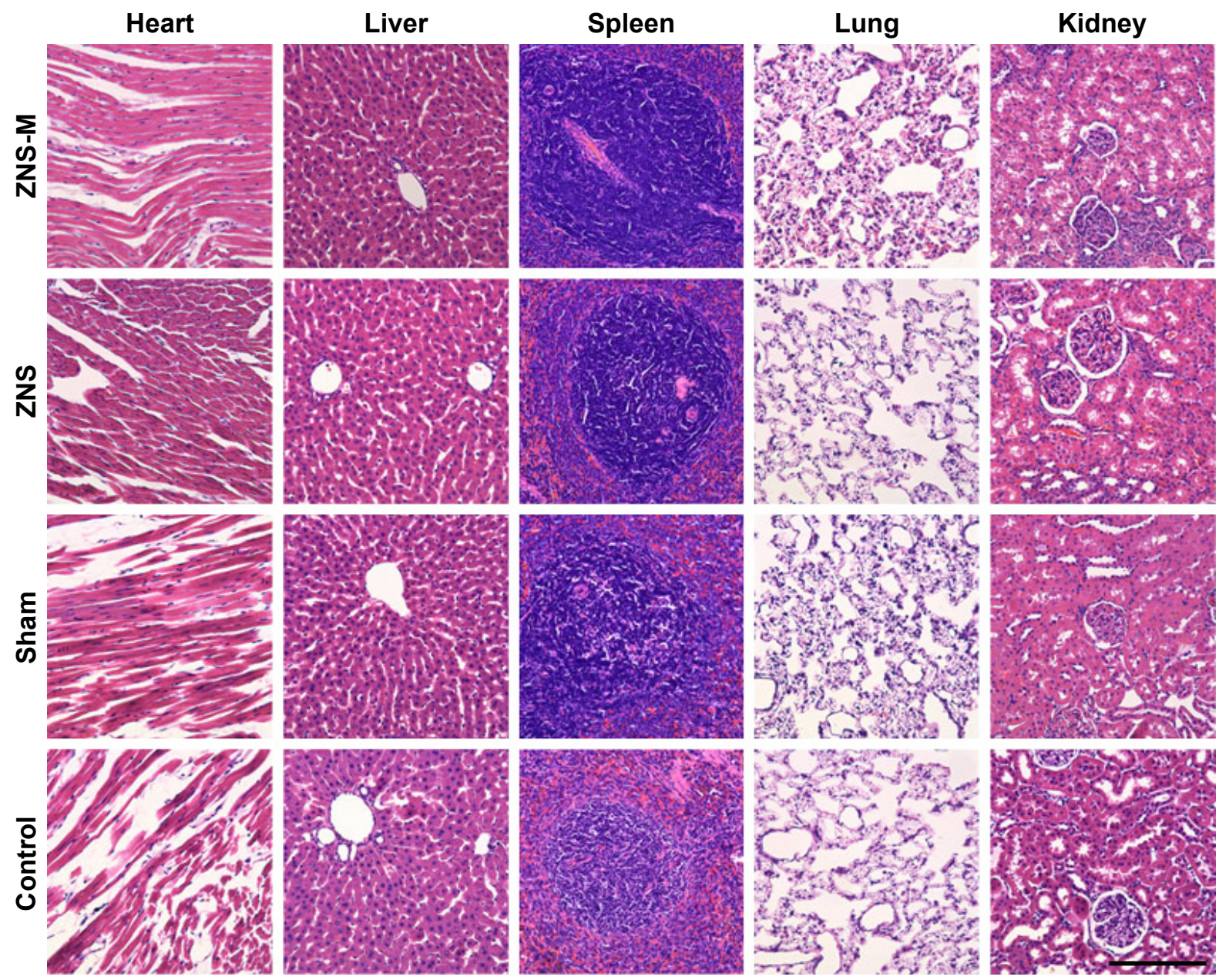

Figure 10 Toxicity evaluation in vivo.

Notes: H\&E staining of SCI rats treated with the ZNS-M, free ZNS and blank M for 4 weeks. Scale bar $=50 \mu \mathrm{m}$.

Abbreviations: H\&E, hematoxylin-eosin; SCl, spinal cord injury; M, micelles; ZNS, zonisamide; ZNS-M, zonisamide micelles.

ZNS-M groups (Figure 7). Enhanced neurite elongation in primary motor neurons and axonal regeneration may partially account for the enhancements of BDNF and Ntrk2 by ZNS, ${ }^{38}$ although the underlying mechanisms remain unknown.

An ideal treatment for inducing axon regeneration in an injured spine should reduce growth-inhibitory factors at the lesion site and be administrable as a medication after the injury. ${ }^{45,46}$ Many antioxidants show promising results in vitro, but their translation in vivo is limited by a short half-life, limited efficiency and metabolism. In this study, the stable ZNS-loaded micelles and free ZNS were administered by injection of the caudal vein. To determine whether ZNS-M could improve the functional outcomes in SCI in animal models, we performed a behavioral study to evaluate the hind-limb functional recovery using the BBB locomotor rating scale. Three days after surgery, the BBB scores began to show differences among the treatment groups, indicating that the function of the hind limbs was gradually recovering. Importantly, the recovered function in the ZNS-M treatment group was better than that of the free ZNS group and the control group 21 days after SCI (Figure 8B). In addition, the results shown by MRI revealed that the atrophied area of the spinal cord in the ZNS-M group (Figure 8Ac) was obviously smaller than the area in the other SCI groups (Figure 8Ab and d). These results signified that ZNS-M treatment could promote motor function recovery after SCI. We believe that ZNS loaded in nanoscale micelles could prevent glomerular filtration, thus extending the retention time of the drug in the blood. Compared to free ZNS, this prolonged effective drug duration of released ZNS may provide better neuroprotective effects in vivo. ${ }^{25,47,48}$

Neuron and axon density are critical indicators of anatomical recovery from SCI. ${ }^{43,49}$ In histopathologic examination, a statistically significant increase of NF was observed in the ZNS-M group (Figure 9). Injury to neurons stimulates a rapid production of superoxide, resulting in cell death. ${ }^{3}$ Thus, inhibition of the oxidase enzyme could result in improvements in functional recovery. However, ZNS-M treatment in vivo did 
not result in a reduction in the number of GFAP that was as significant as the reduction observed in vitro. The reason for this result is unclear, and whether astrocyte scar formation benefits axon regeneration remains controversial. ${ }^{49}$ In addition, the toxicity evaluation demonstrated that the ZNS-M is a safe treatment for injection in vivo (Figure 10).

\section{Conclusion}

In this work, a novel triblock MPEG-PLLA-PTMC copolymer was successfully synthesized. Polymeric micelles loaded with ZNS were prepared and evaluated for the treatment of SCI in vitro and in vivo. The controlled-release ZNS-M were $42.7 \pm 3.2 \mathrm{~nm}$ with good solubility and stability at room temperature. ZNS-M plays a neuron-protective role by antioxidative effect in vivo. In addition, ZNS-M treatment could promote motor function recovery in a hemisection SCI model. Therefore, ZNS-M may be a clinically applicable therapeutic drug for SCI in the future.

\section{Acknowledgments}

This work was financially supported by the National Natural Sciences Foundation of China (81571259) and the National S\&T Major Project (2011ZX09102-001-10).

\section{Disclosure}

The authors report no conflicts of interest in this work.

\section{References}

1. Chiu WT, Lin HC, Lam C, Chu SF, Chiang YH, Tsai SH. Review paper: epidemiology of traumatic spinal cord injury: comparisons between developed and developing countries. Asia Pac J Public Health. 2010; 22(1):9-18.

2. Liu P, Yao Y, Liu MY, et al. Spinal trauma in mainland China from 2001 to 2007: an epidemiological study based on a nationwide database. Spine. 2012;37(15):1310-1315.

3. Bracken MB, Shepard MJ, Holford TR, et al. Administration of methylprednisolone for 24 or 48 hours or tirilazad mesylate for 48 hours in the treatment of acute spinal cord injury. Results of the Third National Acute Spinal Cord Injury Randomized Controlled Trial. National Acute Spinal Cord Injury Study. JAMA. 1997;277(20):1597-1604.

4. Qian T, Guo X, Levi AD, Vanni S, Shebert RT, Sipski ML. High-dose methylprednisolone may cause myopathy in acute spinal cord injury patients. Spinal Cord. 2005;43(4):199-203.

5. Suberviola B, González-Castro A, Llorca J, Ortiz-Melón F, Miñambres E. Early complications of high-dose methylprednisolone in acute spinal cord injury patients. Injury. 2008;39(7):748-752.

6. Snyder EY, Teng YD. Stem cells and spinal cord repair. N Engl J Med. 2012;366(20):1940-1942.

7. Xiong Y, Rabchevsky AG, Hall ED. Role of peroxynitrite in secondary oxidative damage after spinal cord injury. $J$ Neurochem. 2007;100(3): 639-649.

8. Siesjo BK, Bengtosson F. Calcium fluxes, calcium antagonists, and calcium-related pathology in brain ischemia, hypoglycemia, and spreading depression: a unifying hypothesis. J Cereb Blood Flow Metab. 1989;9(2): 127-140.

9. Pellegrini-Giampietro DE, Cherici G, Alesiani M, Carla V, Moroni F. Excitatory amino acid release and free radical formation may cooperate in the genesis of ischemia-induced neuronal damage. $J$ Neurosci. 1990;10(3):1035-1041.
10. Johnstone JT, Morton PD, Jayakumar AR, et al. Inhibition of NADPH oxidase activation in oligodendrocytes reduces cytotoxicity following trauma. PLoS One. 2013;8(11):e80975.

11. Leppik IE. Zonisamide: chemistry, mechanism of action, and pharmacokinetics. Seizure. 2004;13(suppl 1):S5-S9.

12. Sonsalla PK, Wong LY, Winnik B, Buckley B. The antiepileptic drug zonisamide inhibits MAO-B and attenuates MPTP toxicity in mice: clinical relevance. Exp Neurol. 2010;221(2):329-334.

13. Yürekli VA, Gürler S, Nazıroğlu M, Uğuz AC, Koyuncuoğlu HR. Zonisamide attenuates MPP+-induced oxidative toxicity through modulation of $\mathrm{Ca} 2+$ signaling and caspase- 3 activity in neuronal PC12 cells. Cell Mol Neurobiol. 2013;33(2):205-212.

14. Tyler JY, Xu XM, Cheng JX. Nanomedicine for treating spinal cord injury. Nanoscale. 2013;5(19):8821-8836.

15. Raza K, Kumar N, Misra C, et al. Dextran-PLGA-loaded docetaxel micelles with enhanced cytotoxicity and better pharmacokinetic profile. Int J Biol Macromol. 2016;88:206-212.

16. Rezazadeh M, Emami J, Hasanzadeh F, et al. In vivo pharmacokinetics, biodistribution and anti-tumor effect of paclitaxel-loaded targeted chitosanbased polymeric micelle. Drug Deliv. 2016;23(5):1707-1717.

17. Chen WL, Yang SD, Li F, et al. Tumor microenvironment-responsive micelles for pinpointed intracellular release of doxorubicin and enhanced anti-cancer efficiency. Int J Pharm. 2016;511(2):728-740.

18. Duan Y, Wang J, Yang X, Du H, Xi Y, Zhai G. Curcumin-loaded mixed micelles: preparation, optimization, physicochemical properties and cytotoxicity in vitro. Drug Deliv. 2015;22(1):50-57.

19. Pardridge WM. CSF, blood-brain barrier, and brain drug delivery. Expert Opin Drug Deliv. 2016;13(8):1121-1131.

20. Ono K, Hasegawa K, Naiki H, Yamada M. Curcumin has potent antiamyloidogenic effects for Alzheimer's beta-amyloid fibrils in vitro. J Neurosci Res. 2004;75(6):742-750.

21. Chen CL, Chang SF, Lee D, et al. Bioavailability effect of methylprednisolone by polymeric micelles. Pharm Res. 2008;25(1):39-47.

22. Shi Y, Kim S, Huff TB, et al. Effective repair of traumatically injured spinal cord by nanoscale block copolymer micelles. Nat Nanotechnol. 2010;5(1):80-87.

23. Gao X, Wang S, Wang B, et al. Improving the anti-ovarian cancer activity of docetaxel with biodegradable self-assembly micelles through various evaluations. Biomaterials. 2015;53:646-658.

24. Chen B, Bohnert D, Borgens RB, Cho Y. Pushing the science forward: chitosan nanoparticles and functional repair of CNS tissue after spinal cord injury. J Biol Eng. 2013;5:7-9.

25. Wang S, Chen R, Morott J, Repka MA, Wang Y, Chen M. mPEG-bPCL/TPGS mixed micelles for delivery of resveratrol in overcoming resistant breast cancer. Expert Opin Drug Deliv. 2015;12:361-373.

26. Cho Y, Shi R, Borgens R, Ivanisevic A. Repairing the damaged spinal cord and brain with nanomedicine. Small. 2008;4:1676-1681.

27. Kim YT, Caldwell JM, Bellamkonda RV. Nanoparticle-mediated local delivery of Methylprednisolone after spinal cord injury. Biomaterials. 2009;30:2582-2590.

28. Marta S, Andrzej D, Agnieszka A, et al. Lactide/trimethylene carbonate triblock copolymers: controlled sequential polymerization and properties. Polymer. 2016;87:50-63.

29. Chen H, Ruckenstein E. Micellar structures in nanoparticle-multiblock copolymer complexes. Langmuir. 2014;30(13):3723-3728.

30. Chen H, Ruckenstein E. Formation and degradation of multicomponent multicore micelles: insights from dissipative particle dynamics simulations. Langmuir. 2013;29(18):5428-5434.

31. Trinca RB, Abraham GA, Felisberti MI. Electrospun nanofibrous scaffolds of segmented polyurethanes based on PEG, PLLA and PTMC blocks: physico-chemical properties and morphology. Mater Sci Eng C Mater Biol Appl. 2015;56:511-517.

32. Basso DM, Beattie MS, Bresnahan JC. A sensitive and reliable locomotor rating scale for open field testing in rats. J Neurotraum. 1995; 12:1-21.

33. White-Schenk D, Shi R, Leary JF. Nanomedicine strategies for treatment of secondary spinal cord injury. Int J Nanomedicine. 2015; 10:923-938. 
34. Wert KJ, Mahajan VB, Zhang L, et al. Neuroretinal hypoxic signaling in a new preclinical murine model for proliferative diabetic retinopathy. Signal Transduction Targeted Therapy. 2016;1:16005.

35. Jörg Ruschel FH, Flynn KC, Dupraz S, et al. Systemic administration of epothilone B promotes axon regeneration after spinal cord injury. Science. 2015;348(6232):347-352.

36. Hellal F, Hurtado A, Ruschel J, et al. Microtubule stabilization reduces scarring and causes axon regeneration after spinal cord injury. Science. 2011;331(6019):928-931.

37. Bunge MB. Bridging areas of injury in the spinal cord. Neuroscientist. 2001;7(4):325-339.

38. Yagi H, Ohkawara B, Nakashima H, et al. Zonisamide enhances neurite elongation of primary motor neurons and facilitates peripheral nerve regeneration in vitro and in a mouse model. PLoS One. 2015; 10(11):e0142786.

39. Sun Z, Xie H, Tang S, et al. Ultrasmall black phosphorus quantum dots: synthesis and use as photothermal agents. Angew Chem Int Ed Engl. 2015;54(39):11526-11530.

40. Fan R, Tong A, Li X, et al. Enhanced antitumor effects by docetaxel/ LL37-loaded thermosensitive hydrogel nanoparticles in peritoneal carcinomatosis of colorectal cancer. Int J Nanomedicine. 2015; 10:7291-7305.

41. Wang Y, Zhao B, Wang S, et al. Formulation and evaluation of novel glycyrrhizic acid micelles for transdermal delivery of podophyllotoxin. Drug Deliv. 2016;23(5):1623-1635.
42. Markowitz AJ, White MG, Kolson DL, Jordan-Sciutto KL. Cellular interplay between neurons and glia: toward a comprehensive mechanism for excitotoxic neuronal loss in neurodegeneration. Cellscience. 2007;4(1):111-146.

43. Zheng LT, Ock J, Kwon B-M, Suk K. Suppressive effects of flavonoid fisetin on lipopolysaccharide-induced microglial activation and neurotoxicity. Int Immunopharmacol. 2008;8(3):484-494.

44. GhoshMitra S, Diercks DR, Mills NC, Hynds DL, Ghosh S. Role of engineered nanocarriers for axon regeneration and guidance: current status and future trends. Adv Drug Deliv Rev. 2012;64(1):110-125.

45. Ruschel J, Hellal F, Flynn KC, et al. Axonal regeneration. Systemic administration of epothilone B promotes axon regeneration after spinal cord injury. Science. 2015;348(6232):347-352.

46. Shao J, Xie H, Huang H, et al. Biodegradable black phosphorus-based nanospheres for in vivo photothermal cancer therapy. Nat Commun. 2016;7:12967.

47. Zheng S, Gao X, Liu X, et al. Biodegradable micelles enhance the antiglioma activity of curcumin in vitro and in vivo. Int J Nanomedicine. 2016;11:2721-2736.

48. Zhao Y, Wang H, Huang H, et al. Surface coordination of black phosphorus for robust air and water stability. Angew Chem Int Ed Engl. 2016;55(16):5003-5007.

49. Anderson MA, Burda JE, Ren Y, et al. Astrocyte scar formation aids central nervous system axon regeneration. Nature. 2016;532(7598): 195-200.
International Journal of Nanomedicine

\section{Publish your work in this journal}

The International Journal of Nanomedicine is an international, peerreviewed journal focusing on the application of nanotechnology in diagnostics, therapeutics, and drug delivery systems throughout the biomedical field. This journal is indexed on PubMed Central, MedLine, CAS, SciSearch $®$, Current Contents ${ }^{\circledR} /$ Clinical Medicine,

\section{Dovepress}

Journal Citation Reports/Science Edition, EMBase, Scopus and the Elsevier Bibliographic databases. The manuscript management system is completely online and includes a very quick and fair peer-review system, which is all easy to use. Visit http://www.dovepress.com/ testimonials.php to read real quotes from published authors. 